\title{
ORIGINAL
}

\section{PREVALENCIA Y DISTRIBUCIÓN GEOGRÁFICA DEL CÁNCER EN EL ÁREA DE SALUD DE GUADALAJARA (*)}

\begin{abstract}
Alonso Gordo José Ma (1), Jiménez del Val Dolores (1), Palacios Rojo Juan José (2), Royo Sánchez Carlos (3), Urbina Torija Juan Román (1), Santiago González Catalina (1), Bárcena Marugán Aurora (1) Grupo PREVALCAN de Guadalajara.
\end{abstract}

(1) Gerencia de Atención Primaria de Salud. Guadalajara.

(2) Hospital General de Guadalajara.

(3) Servicio de Salud de Castilla-La Mancha.

\section{RESUMEN}

Fundamento: La prevalencia total de cáncer oscila entre un 2 y un $3 \%$ de la población. Algunos datos sugieren diferencias en función del medio urbano o rural, de otros factores geográficos, y en el entorno de las centrales nucleares. El objetivo de este trabajo es conocer dichas prevalencias y su distribución en las diferentes zonas geográficas del Area de Salud de Guadalajara.

Métodos: Estudio de prevalencia de punto, en 1999, mediante revisión sistemática de casos en fuentes primarias y secundarias del Area de Salud de Guadalajara. Se incluyen tumores malignos invasivos en mayores de 14 años. Se analiza la distribución general y específica en función del lugar de residencia, comarca, y proximidad a las centrales nucleares. Se calculan prevalencias crudas y ajustadas/100.000 h y razón de prevalencia con IC al $95 \%$.

Resultados: Se detectaron 2.717 casos (prevalencia cruda: $2034,6 / 10^{5}$ ), repartidos al $50 \%$ entre el medio urbano y el rural. La prevalencia ajustada (a población mundial) es de $1295,2 / 10^{5}$, mayor en el medio urbano $\left(1479,9 / 10^{5}\right)$ que en el rural $\left(1136,3 / 10^{5}\right)$. En relación con us comarcas geogránicas únicamente el cáncer de tiroi des es más prevalente en mujeres en las zonas más deprimidas y des es más prevalente en mujeres en
montañosas, si bien con baja casuística.

Conclusiones: Las prevalencias de cáncer encontradas son similares a las publicadas y, tras ajuste por edad, son mayores en el medio urbano que en el rural. Las diferencias entre comarcas se relacionan con las características demográficas, geográficas y el carácter urbano.

Palabras clave: Neoplasias. Prevalencia. Registros médicos orientados a problemas. Hábitat. Reactores Nucleares. Atención Primaria de Salud.

Correspondencia:

José $\mathrm{M}^{\mathrm{a}}$ Alonso Gordo

Gerencia de Atención Primaria

C/Ferial, 31,

19002. Guadalajara (España).

Correo electrónico: jmalonso@sescam.org

\section{ABSTRACT}

\section{Cancer Prevalence and Geographical Distribution in the Guadalajara Healthcare District, Spain}

Background: The total cancer prevalence falls within the range of $2 \%-3 \%$ of the population. Some data suggests differences in terms of whether the environment is urban or rural, other geographical factors and in the area surounding nuclear power pic phical facty and bution thereof in the different geographical areas of the Guadalajara Healthcare District.

Methods: Point prevalence study in 1999 based on a systematic review of cases at primary and secondary sources in the Guadalajar Health District, including invasive malignant tumors among individuals over age 14. The overall and specific distribution in terms of the place of residence, region and proximity to nuclear power plants is analyzed, raw and adjusted prevalences/100,000 inhabitants and is analyzed, raw and adju

Results: A total of 2,717 cases were detected (raw prevalence: $2034.6 / 10^{5}$ ), there being a $50 \%-50 \%$ ratio between the urban and rural environments. The adjusted prevalence (worldwide population) is $1295.2 / 10^{5}$, being greater in the urban environment $\left(1479.9 / 10^{5}\right)$ than in the rural environment $\left(1136.3 / 10^{5}\right)$. With regard to the geographic regions, solely thyroid cancer is more prevalent among women in the most depressed, mountainous areas, although involving a low-level casuistic.

Conclusions: The cancer prevalences found are similar to those published and, following an age-based adjustment, are greater in the urban than in the rural environment. The differences among regions are related to the demographic and geographical characteristics and to the environment being urban.

Key words: Neoplasms. Prevalence. Habitat. Nuclear reactors. Medical records, problem-oriented. Primary Health.

(1) Trabajo financiado con la Ayuda del Fondo de Investigaciones Sanitarias 99/0250. 


\section{INTRODUCCIÓN}

El número total de personas que han padecido cáncer en algún momento de su vida es una cifra elevada. Sabemos que en el año 2000 se produjeron en España unos 143.000 casos de cáncer de nuevo diagnóstico ${ }^{1} \mathrm{y}$ alrededor de 95.000 muertes por este motivo $(26 \% \text { del total de fallecimientos })^{2}$, siendo superior el número de personas que fallecen con un diagnóstico de cáncer; en dicho año se produjeron un total de 259.098 altas hospitalarias con diagnóstico de neoplasia ${ }^{3}$.

El conocimiento del número de personas con dicho diagnóstico en sus antecedentes es un dato valioso bajo el punto de vista asistencial y de la planificación sociosanitaria ${ }^{4,5}$ y ha sido abordado en diversas ocasiones, aunque muy escasamente en nuestro país. Estudios realizados en otros países, en diversas épocas y con distintas metodologías, estiman que la prevalencia total de cáncer oscila entre un 2 y un $3 \%$ de la población ${ }^{6-10}$. Según cálculos de la Agencia Internacional de Investigación sobre el cáncer ${ }^{1}$, se estimaba que en España en el año 2000 vivían 396.422 personas con cáncer diagnosticado en los últimos 5 años; esta cifra supone una prevalencia a 5 años de alrededor del $1 \%$, lo que significa aproximadamente el $50 \%$ de la prevalencia total.

Aunque no existen razones específicas generalizables para esperar un comportamiento distinto según los lugares de residencia, se han publicado datos ajustados de mayor incidencia y prevalencia de cáncer de la mayoría de las localizaciones en el medio urbano y grandes ciudades ${ }^{11,12}$. Se conocen, asimismo, diferencias en función de la residencia en medio rural, atribuibles, sobre todo, a la exposición a la radiación solar y a la mayor o menor actividad laboral de riesgo, industrial o agrícola ${ }^{13,14}$. Otros estudios en nuestro país han apuntado diferencias en la incidencia ${ }^{15-17}$ o en la mortalidad por cáncer, sobre todo de tipo hematológico, en relación con la proximidad a Centrales Nucleares ${ }^{18,19}$.
Datos del Hospital General de Guadalajara indican que los ingresos por cáncer en 1999 supusieron 16.929 días de estancia, siendo el número de nuevos casos en dicho año de 586 y el de fallecimientos de 419 $(26,3 \%)$. Estudios previos en nuestra Area de Salud (AS) apuntan también a diferencias en función de diversos factores geográficos en la distribución de dichos ingresos ${ }^{15,16}$. Con el propósito de conocer la prevalencia real de cáncer en adultos y mejorar la información epidemiológica sobre esta enfermedad en nuestra AS de Guadalajara, realizamos un estudio cuya metodología y resultados iniciales fueron publicados recientemente ${ }^{20}$. En función de los datos de incidencia y mortalidad, podríamos estimar entre 3.000 y 4.000 el número total de personas vivas diagnosticadas de cáncer en nuestra AS, cifra que fue confirmada en nuestro estudio; nos proponemos en este trabajo presentar los datos de prevalencia según el lugar de residencia, en función del medio urbano o rural, comarcas del Area, zonas próximas a las Centrales Nucleares y de cada Zona Básica de Salud (ZBS).

\section{MATERIAL Y MÉTODOS}

Ambito y tipo de estudio. Realizamos el estudio en el AS de Guadalajara, una de las siete en que está dividida sanitariamente la Comunidad Autónoma de Castilla-La Mancha y que coincide, en líneas generales, con la provincia; existen $28 \mathrm{ZBS}$, repartidas en las cuatro comarcas naturales además de la ciudad: Alcarria, Campiña, Señorío de Molina y Serranía (figura 1). El diseño corresponde a un estudio descriptivo, transversal de prevalencia de punto, realizado a mediados de 1999.

La población del AS se agrupa en un 54\% en la capital (con 69.000 h) y Azuqueca de Henares (con 20.000 h), a las que hemos considerado zona urbana, y el resto en un entorno rural muy disperso de unos 400 núcleos poblacionales; la población es enve- 


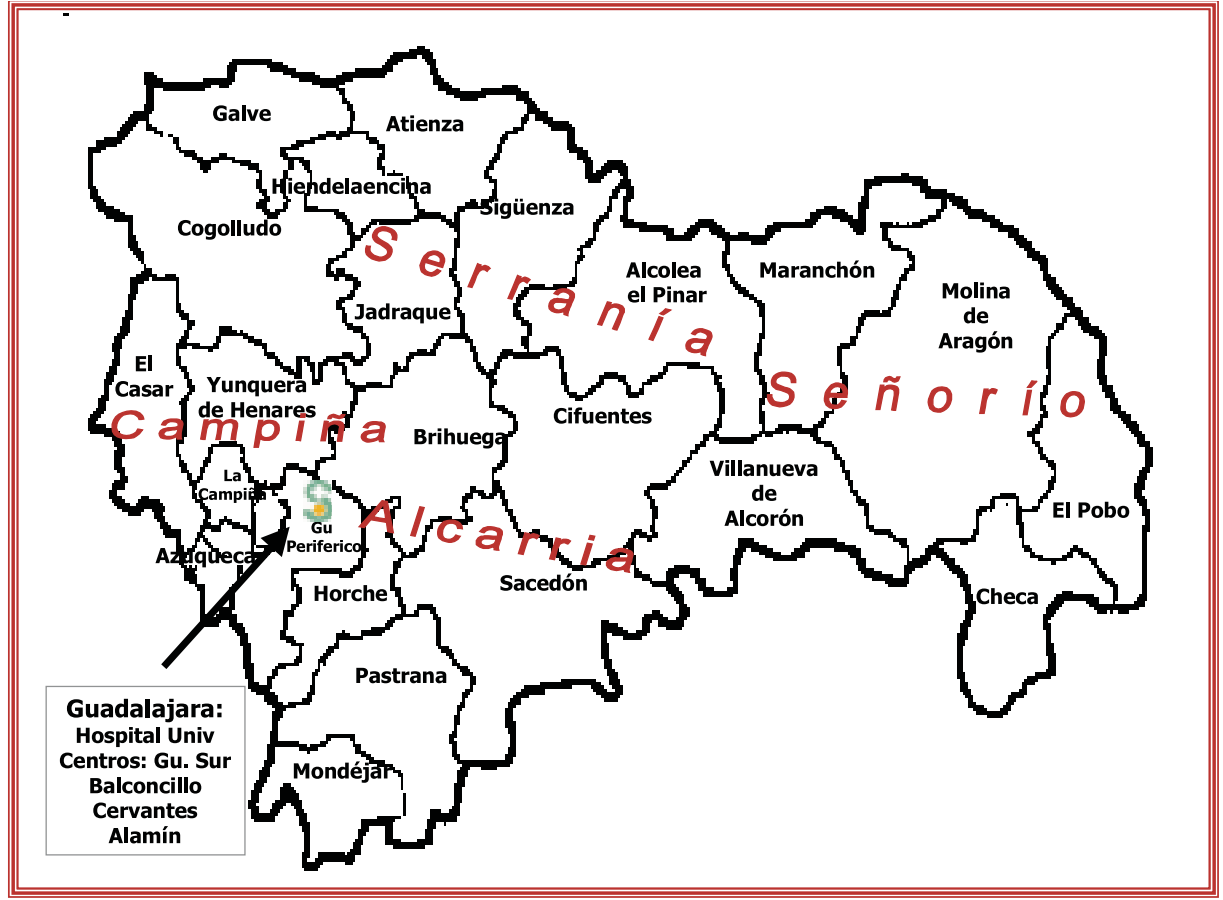

jecida, con una proporción mayores de 64 años/menores de 15 de 1,60, aunque en las Comarcas de la Serranía y Señorío esta cifra es superior a 4 . Un $15,5 \%$ residen dentro de un diámetro de $30 \mathrm{Km}$ alrededor de las centrales nucleares de Trillo y Zorita de los Canes (figura 1), configurando la zona del Plan de Emergencia Nuclear de Guadalajara $(\text { PENGUA })^{21}$.

La población estudiada corresponde a mayores de 14 años con Tarjeta Individual Sanitaria (TIS) en Junio de 1999 (133.539 personas). La asistencia sanitaria es adscrita mediante dicha tarjeta a los 28 Equipos de Atención Primaria (EAP), sobre los que realizamos el estudio. La asistencia hospitalaria es prestada por un único Hospital General público en la ciudad de Guadalajara y los hospitales de referencia de Madrid y Areas limítrofes, a los que se accede mediante la correspondiente Orden de Asistencia Interprovincial.

Sujetos. Se ha considerado paciente con cáncer prevalente, tal y como se ha publicado previamente ${ }^{20}$ a todo persona que, figurando en la base de datos de TIS del AS en el momento del corte, disponía de un diagnóstico previo de cáncer invasivo en Atención Primaria o especializada, según códigos 140 a 208 de la Clasificación Internacional de Enfermedades (CIE-9) ${ }^{22,23}$. Se incluyeron los casos de los que existía constancia de informe anatomopatológico en cualquier fuente de datos, diagnóstico de enfermedad maligna en los Datos Básicos de los Servicios de Admisión hospitalarios confirmado por alguna de las otras fuentes, o diagnóstico exclusivamente clínico, analítico o de imagen. 
Datos recogidos. Se utilizó un cuestionario que incluía los datos de identificación personal, de residencia, asistenciales y del tumor: localización nominal y mediante código CIE-9, tipo histológico, fecha, lugar y métodos de diagnóstico.

Como fuente de información se han utilizado los datos proporcionados por los Médicos de Familia del Sistema sanitario público, bajo la coordinación de un responsable del estudio en cada EAP. Como fuentes secundarias se han revisado, secuencialmente, la información existente en el Registro Provincial de Tumores, Sistemas de Información Hospitalaria, Residencias de Ancianos y enfermos crónicos, Clínicas privadas, Registro Civil de mortalidad y Ordenes Nacionales de Asistencia. Los datos incompletos se confirmaron en el resto de las fuentes y en el Servicio de Anatomía Patológica del Hospital General. Se establecieron criterios comunes de inclusión y exclusión y criterios internos de incompatibilidad.

Análisis de datos. Se ofrecen datos de edad de diagnóstico, edad actual y tiempo de evolución del tumor, con comparación de medias y desviación estándar para cada grupo de estudio en función del lugar de residencia en el momento del corte. Los datos de prevalencia se presentan en números absolutos y porcentuales, y en prevalencia/100.000 habitantes, crudas y ajustadas por el método directo a población mundial (PAM) ${ }^{23}$ y a población total española en el año 1998 (PAE). Para las comparaciones entre las diversas comarcas utilizamos el ajuste a población española, dado que es más parecida a la de Guadalajara, produciendo menor distorsión de los datos que la mundial.

Se ha calculado, asimismo, la razón de prevalencia entre los grupos estudiados sobre los casos teóricos tras el ajuste de tasas; dada la distribución asimétrica de los riesgos (el valor mínimo de la razón es cero y el máximo puede ser infinito), los intervalos de confianza al $95 \%$ se calcularon mediante el error estándar del logaritmo natural de dicha razón ${ }^{23}$. En todo caso, dado el tamaño de la población, dicho cálculo ofrece datos sensiblemente iguales a los de la Odd Ratio de prevalencias y su prueba de $\mathrm{Chi}^{2}$. Los datos se analizan y comparan en función del medio urbano o rural (con límite en $10.000 \mathrm{~h}$ ), comarcas naturales y zona de emergencia nuclear definida por debajo de los $30 \mathrm{Km}$ desde las centrales de Zorita y Trillo. Se presentan también datos para cada ZBS con sus límites de confianza al 95\%.

\section{RESULTADOS}

Se encontraron, en mayores de 14 años, un total de 2.717 neoplasias distintas, de las que existía constancia de confirmación histológica en el 93,8\% de los casos. La prevalencia cruda es de 2034,6/100.000h, y la ajustada a población mundial de 1295,2. En la tabla 1 se presentan los datos generales y las diferencias entre los medios urbano y rural y por sexo. Podemos comprobar una edad media en el momento del estudio y de diagnóstico, superior en el medio rural y una edad de diagnóstico menor en el caso de las mujeres, con mayor tiempo de evolución. En relación con las comarcas estudiadas (geográficas y de pertenencia al PENGUA) estos datos generales no ofrecen diferencias que no estén en relación con la pertenencia al medio urbano o rural.

Los valores de prevalencia cruda para el medio urbano son: $1893,2 / 10^{5}$ y en el medio rural $2198,3 / 10^{5}$, si bien al ajustar por edad las proporciones se invierten $\left(1479,8 / 10^{5} \mathrm{y}\right.$ $1136,3 / 10^{5}$ respectivamente, ajustando a población mundial). La tabla 2 nos muestra la prevalencia/100.000 h cruda y ajustada a población española (PAE), distribuida en función del medio urbano y rural, y la razón de prevalencias ajustadas. Como podemos comprobar, las cifras ajustadas y el riesgo son superiores en casi todas las localizaciones para el medio urbano que para el rural. 
Tabla 1

Datos generales de los casos de cáncer prevalente

\begin{tabular}{|c|c|c|c|c|c|c|}
\hline & MEDIO & URBANO & MEDI & RURAL & \multirow{2}{*}{\multicolumn{2}{|c|}{ TOTAL: 2717}} \\
\hline & \multicolumn{2}{|c|}{$(\mathrm{N}=1355)$} & \multicolumn{2}{|c|}{$(\mathrm{N}=1362)$} & & \\
\hline & Hombres & Mujeres & Hombres & Mujeres & Hombres & Mujeres \\
\hline Casos $(\%)$ & $725(53,5)$ & $630(46,5)$ & $818(60,1)$ & $544(39,9)$ & $1543(56,8)$ & $1174(43,2)$ \\
\hline Edad actual (DE) & $67,7(14,8)$ & $64,5(15,7)$ & $71,0(12,7)$ & $69,0(14,0)$ & $69,5(13,8)$ & $66,5(15,1)$ \\
\hline Edad de diagnóstico (DE) & $62,9(15,4)$ & $57,7(16,1)$ & $66,4(13,6)$ & $63,3(14,7)$ & $64,8(14,6)$ & $60,3(15,7)$ \\
\hline Evolución (meses) (DE) & $57,9(57,0)$ & $77,2(82,4)$ & $56,6(62,9)$ & $67,3(76,3)$ & $57,2(60,2)$ & $72,6(79,7)$ \\
\hline
\end{tabular}

Tabla 2

Prevalencia de cáncer/100.000 en función del medio y sexo

\begin{tabular}{|c|c|c|c|c|c|c|c|c|c|c|}
\hline \multirow[b]{3}{*}{ LOCALIZACIÓN } & \multicolumn{5}{|c|}{ HOMBRES } & \multicolumn{5}{|c|}{ MUJERES } \\
\hline & \multicolumn{2}{|c|}{ URBANO } & \multicolumn{2}{|c|}{ RURAL } & \multirow[b]{2}{*}{$\begin{array}{c}\text { Razón } \\
\text { (IC 95\%) }\end{array}$} & \multicolumn{2}{|c|}{ URBANO } & \multicolumn{2}{|c|}{ RURAL } & \multirow[b]{2}{*}{$\begin{array}{c}\text { Razón } \\
\text { (IC 95\%) }\end{array}$} \\
\hline & $\mathrm{PC}$ & PAE & $\mathrm{PC}$ & PAE & & $\mathrm{PC}$ & PAE & $\mathrm{PC}$ & PAE & \\
\hline TODOS & 2067 & 2441,6 & 2560,2 & 1798 & $\begin{array}{c}1,36 \\
1,22-1,51\end{array}$ & 1726,6 & 1798,8 & 1812,9 & 1322,9 & $\begin{array}{c}1,36 \\
1,20-1,54\end{array}$ \\
\hline Glándula prostática & 348,3 & 426,8 & 497 & 288,1 & $\begin{array}{c}1,48 \\
(1,14-1,92)\end{array}$ & - & - & - & - & \\
\hline Mama & NR & $\mathrm{NR}$ & NR & NR & - & 681,2 & 724,4 & 683,2 & 522 & $\begin{array}{c}1,39 \\
1,14-1,69 \\
\end{array}$ \\
\hline Cuerpo de útero & - & - & - & - & - & 150,5 & 170,3 & 140 & 104,2 & $\begin{array}{c}1,63 \\
1,06-2,51\end{array}$ \\
\hline Ovario & - & - & - & - & - & 84,8 & 94,2 & 93,3 & 78,0 & $\begin{array}{c}1,21 \\
0,71-2,04\end{array}$ \\
\hline Vejiga Urinaria & 396,8 & 472,1 & 375,6 & 247,7 & $\begin{array}{c}1,91 \\
(1,46-2,49)\end{array}$ & 60,2 & 61,9 & 56,7 & 29,2 & $\begin{array}{c}2,12 \\
0,97-4,62\end{array}$ \\
\hline Colon y recto & 262,7 & 318,1 & 366,2 & 267,6 & $\begin{array}{c}1,19 \\
(0,90-1,58)\end{array}$ & 191,5 & 189,3 & 233,3 & 135,3 & $\begin{array}{c}1,39 \\
0,95-2,06\end{array}$ \\
\hline Estómago & 131,3 & 150,5 & 150,2 & 103,8 & $\begin{array}{c}1,45 \\
(0,94-2,23)\end{array}$ & 82,1 & 81 & 93,9 & 56,1 & $\begin{array}{c}1,44 \\
0,79-2,63\end{array}$ \\
\hline $\begin{array}{l}\text { Tráquea, bronquios y } \\
\text { pulmón. }\end{array}$ & 197,0 & 232,5 & 212,8 & 153,3 & $\begin{array}{c}1,52 \\
(1,06-2,16)\end{array}$ & NR & NR & NR & NR & 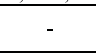 \\
\hline Laringe & 134,2 & 156,1 & 190,9 & 137,9 & $\begin{array}{c}1,13 \\
(0,76-1,68)\end{array}$ & NR & NR & NR & NR & $\cdot$ \\
\hline \begin{tabular}{|l|}
$\begin{array}{l}\text { Riñóón/órganos } \\
\text { urinarios }\end{array}$ \\
\end{tabular} & 105,6 & 123,4 & 100,2 & 69,1 & $\begin{array}{c}1,78 \\
(1,07-2,98)\end{array}$ & 62,9 & 63,9 & 20 & 17,9 & $\begin{array}{c}3,57 \\
1,40-9,12\end{array}$ \\
\hline Linfoma No Hodgkin & 48,5 & 52,5 & 87,6 & 71,4 & $\begin{array}{c}0,73 \\
(0,39-1,36)\end{array}$ & 21,9 & 24,5 & 46,7 & 30,5 & $\begin{array}{c}0,8 \\
0,32-2,02 \\
\end{array}$ \\
\hline Glándula Tiroides & 17,1 & 20,9 & 15,7 & 13,4 & $\begin{array}{c}1,56 \\
(0,47-5,14)\end{array}$ & 73,9 & 72,9 & 73,3 & 75,5 & $\begin{array}{c}1,01 \\
0,57-1,77 \\
\end{array}$ \\
\hline Leucemia Linfoide & 28,5 & 37,6 & 50,1 & 33,3 & $\begin{array}{c}1,13 \\
(0,5-2,53)\end{array}$ & 41 & 40,4 & 36,7 & 20,8 & $\begin{array}{c}1,94 \\
0,76-4,95 \\
\end{array}$ \\
\hline Labio (excluida piel) & 51,4 & 63,3 & 87,6 & 58 & $\begin{array}{c}1,09 \\
(0,59-2,02)\end{array}$ & NR & NR & NR & NR & - \\
\hline Melanoma de la piel & 22,8 & 24,8 & 50,1 & 38,6 & $\begin{array}{c}0,64 \\
(0,27-1,53) \\
\end{array}$ & 21,9 & 21,9 & 53,3 & 45,6 & $\begin{array}{c}0,48 \\
0,20-1,15 \\
\end{array}$ \\
\hline
\end{tabular}

P.C: Prevalencia cruda/100.000 h. PAE: Prevalencia ajustada a población española en 1998. Razón IC 95\%: Razón de prevalencia ajustada a población española con intervalo de confianza al 95\%. NR: No reseñable

Las prevalencias globales o específicas de las localizaciones más frecuentes de cáncer en las diversas comarcas del Area Sanitaria quedan reflejadas en la tabla 3. En ella podemos comprobar que las diferencias en las prevalencias crudas se amortiguan de modo importante al realizar el ajuste por edad, no siendo significativa la diferencia entre las diversas comarcas pero sí entre la ciudad y cualquiera de ellas (la razón de prevalencia global de cáncer más baja tras el ajuste por edad, corresponde a la comparación entre 
Figura 2

Prevalencias ajustadas/100.000 h por Zonas básicas de Salud (IC 95\%)

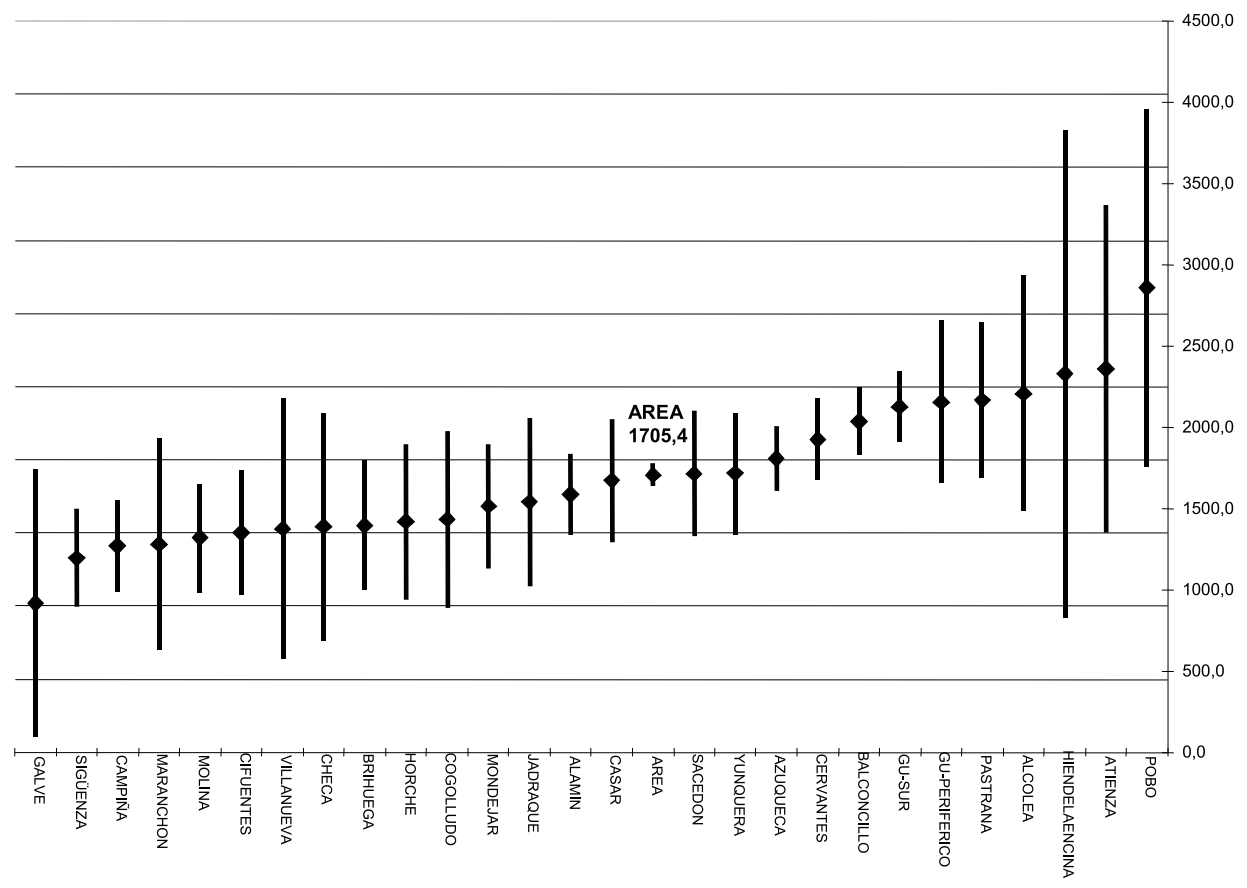

ciudad y Campiña, con cifras de 1,17 e IC al $95 \%$ de 1,06 a 1,30$)$.

En cuanto a las localizaciones específicas analizadas globalmente o distribuidas por sexo, las diferencias entre comarcas no alcanzan valores importantes, situándose en cifras bajas tanto el tamaño poblacional como la casuística. Unicamente son destacables, como hemos comentado, las diferencias entre las zonas urbanas y rurales, y, de modo específico, tras el ajuste poblacional, hemos encontrado diferencias en el cáncer de tiroides en mujeres en el Señorío de Molina (PAE 200/10 $0^{5}$ h) y Serranía (PAE $126 / 10^{5}$ ) frente a las demás comarcas (PAE entre 50 y $75 / 10^{5}$ ).

En la figura 2 podemos ver la distribución de prevalencia por $100.000 \mathrm{~h}$, y sus límites de confianza al $95 \%$, de cada una de las Zonas Básicas de Salud, una vez ajustadas a población española; destacamos el valor medio del área $(1705,4 / 100.000)$ y los valores con intervalos más estrechos de las zonas urbanas (Alamín, Azuqueca, Balconcillo, Cervantes y Gu-Sur); los extremos corresponden, precisamente, a las ZBS de menor población y con límites de confianza más abiertos.

Las cifras globales o específicas en función de las zonas de influencia de las centrales nucleares quedan expuestas en la tabla 4, donde se pueden apreciar los valores de la Razón de Prevalencias ajustadas a población española. Sólo presentamos datos comparativos de la zona rural, ya que no hay zona urbana dentro del ámbito de influencia del PENGUA. Como podemos observar las prevalencias globales o específicas son similares en ambas zonas y la diferencia de riesgos solo alcanza valores discretamente significativos en el caso del cáncer broncopulmonar. 
Tabla 3

Prevalencia de cáncer/100.000 h por comarcas. Ambos sexos

\begin{tabular}{|c|c|c|c|c|c|c|c|c|c|c|}
\hline & \multicolumn{5}{|c|}{ PREVALENCIA CRUDA } & \multicolumn{5}{|c|}{$\begin{array}{c}\text { PREVALENCIA AJUSTADA } \\
\text { (Pob española)) }\end{array}$} \\
\hline & 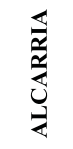 & 空 & 穵 & 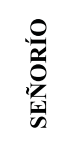 & $\frac{\nwarrow}{\frac{\pi}{\pi}}$ & 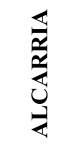 & 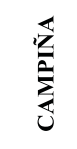 & 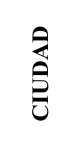 & 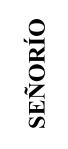 & 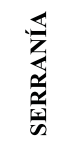 \\
\hline TODOS & 2455 & 1623 & 1956 & 2296 & 2423 & 1676 & 1741 & 2038 & 1543 & 1460 \\
\hline $\begin{array}{l}\text { Mama } \\
\text { (Mujeres) }\end{array}$ & 637,5 & 615,3 & 654,4 & 1065 & 891,5 & 517,7 & 684,8 & 702,7 & 658,1 & 744,1 \\
\hline Próstata (Hombres) & 611,5 & 337,6 & 353,8 & 471,8 & 488,8 & 341,5 & 431,8 & 400,2 & 248,6 & 276 \\
\hline $\begin{array}{l}\text { Vejiga } \\
\text { Urinaria }\end{array}$ & 238,3 & 173,8 & 246,9 & 184,9 & 230 & 227,2 & 182,8 & 257,2 & 114,2 & 112,6 \\
\hline $\begin{array}{l}\text { Colon } \\
\text { y recto }\end{array}$ & 329,1 & 223,5 & 238 & 369,8 & 262,8 & 219,2 & 237,4 & 246,9 & 235 & 143,8 \\
\hline Estómago & 151,3 & 52,7 & 117,2 & 206,4 & 156,1 & 93,6 & 58,9 & 117,2 & 83,3 & 90,6 \\
\hline $\begin{array}{l}\text { Tráquea, bronquios } \\
\text { y pulmón }\end{array}$ & 158,9 & 93,1 & 95,9 & 77 & 106,8 & 119 & 101,7 & 101,9 & 39,1 & 59,6 \\
\hline Laringe & 128,6 & 71,4 & 67,5 & 92,5 & 82,1 & 76,6 & 79,4 & 72,6 & 66,7 & 54 \\
\hline $\begin{array}{l}\text { Cuerpo de } \\
\text { Útero (Mujeres) }\end{array}$ & 171 & 100,5 & 159,3 & 129,1 & 154,3 & 129,2 & 118,6 & 180,2 & 99,3 & 115,1 \\
\hline $\begin{array}{l}\text { Riñón y otros órganos } \\
\text { urinarios }\end{array}$ & 68,1 & 46,6 & 92,4 & 30,8 & 90,4 & 44,1 & 52,7 & 97,4 & 28,3 & 55,7 \\
\hline Linfoma no Hodgkin & 87 & 43,4 & 30,2 & 61,6 & 73,9 & 69,5 & 73,6 & 32 & 44 & 51,1 \\
\hline
\end{tabular}

\section{DISCUSION}

La prevalencia de cáncer es un aspecto escasamente estudiado en nuestro país y los datos publicados, de valor limitado. Nuestro estudio es el único realizado sobre un ámbito poblacional amplio y con datos reales. Avellana $^{24}$ en 1992 recoge los datos de tumores registrados en un Centro de Salud urbano, pudiendo estimarse sus cifras de prevalencia en 570/100.000 h. Botella, por su parte, realiza su estudio en una población y sistema asistencial específico (Instituto Social de las Fuerza Armadas), con cifras de 940 casos $/ 100.000 \mathrm{~h}^{25}$.
Los primeros estudios de base poblacional sobre prevalencia de cáncer corresponden a los países nórdicos, oscilando sus tasas crudas en los años ochenta entre 1752 y 1840/ 100.000 para hombres y entre 2659 y $2808 /$ 100.000 para mujeres ${ }^{11,12,26}$ y ofreciendo también datos diferenciados para las grandes ciudades. Estudios bastante definitivos en los últimos años son los realizados en Italia por Micheli y cols ${ }^{27,28}$, cuyas tasas crudas, a partir de los registros italianos de tumores, son de $2237,9 / 100.000$ para hombres y de 3097,4 para mujeres; dentro de estos estudios las diferencias entre las diversas comarcas italianas llegan a ser muy importantes. Recientemente, 
Tabla 4

Prevalencia de cáncer/100.000 h. en función de pertenencia al PENGUA

\begin{tabular}{|c|c|c|c|c|c|}
\hline \multirow{2}{*}{ LOCALIZACIÓN } & \multicolumn{2}{|c|}{ Zona Rural PENGUA } & \multicolumn{2}{|c|}{$\begin{array}{c}\text { Zona Rural } \\
\text { NO PENGUA } \\
\end{array}$} & \multirow{2}{*}{$\begin{array}{l}\text { Razón de PAE } \\
\text { (I.C. 95\%) }\end{array}$} \\
\hline & PC & PAE & PC & PAE & \\
\hline TODOS & 2409,4 & 1588,4 & 2063,5 & 1492,2 & $1,06(0,93-1,21)$ \\
\hline Mama (Mujeres) & 646,8 & 510,2 & 692,2 & 524 & $0,97(0,70-1,35)$ \\
\hline Próstata (Hombres) & 606,1 & 325,7 & 441,6 & 269,2 & $1,21(0,80-1,84)$ \\
\hline Colon y recto & 280 & 192,1 & 334 & 212,1 & $0,91(0,62-1,31)$ \\
\hline Vejiga Urinaria & 240 & 140 & 206,3 & 130,9 & $1,07(0,68-1,67)$ \\
\hline Cuerpo de útero (Mujeres) & 173,8 & 129,1 & 117,1 & 88,7 & $1,46(0,71-2,97)$ \\
\hline Estómago & 150,6 & 90,8 & 105,6 & 71,7 & $1,27(0,71-2,25)$ \\
\hline Tráquea, bronquios y pulmón & 141,2 & 107 & 81,1 & 53,7 & $1,99(1,11-3,58)$ \\
\hline Ovario (Mujeres) & 96,5 & 89 & 86,5 & 69,5 & $1,28(0,56-2,95)$ \\
\hline Riñón y otros órganos urinarios & 80 & 51,1 & 51,6 & 38,7 & $1,32(0,61-2,86)$ \\
\hline Laringe & 108,2 & 62,9 & 93,3 & 70,4 & $0,89(0,47-1,71)$ \\
\hline Glándula Tiroides & 23,5 & 22,9 & 51,6 & 49,7 & $0,46(0,17-1,24)$ \\
\hline Linfoma No Hodgkin & 94,1 & 67,7 & 54 & 43,5 & $1,56(0,78-3,12)$ \\
\hline Leucemia Linfoide & 32,9 & 23,3 & 49,1 & 30 & $0,78(0,27-2,21)$ \\
\hline Encéfalo & 23,5 & 18,4 & 19,7 & 17,7 & $1,04(0,30-3,56)$ \\
\hline Melanoma de la piel & 42,4 & 23,5 & 56,5 & 52 & $0,46(0,15-1,28)$ \\
\hline Leucemia Mieloide & 9,4 & 5,4 & 24,6 & 21,9 & $0,25(0,04-1,72)$ \\
\hline Mieloma & 14,1 & 7,7 & 31,9 & 23,2 & $0,33(0,6-1,74)$ \\
\hline Enfermedad de Hodgkin & 28,2 & 31,1 & 24,6 & 27,9 & $1,11(0,43-2,91)$ \\
\hline
\end{tabular}

PC: Prevalencia cruda. PAE: Prevalencia ajustada a población española en 1998. Razón de PAE: Razón de prevalencias ajustadas a población española con intervalo de confianza al $95 \%$.

el estudio EUROPREVAL ${ }^{29}$, con datos de 1992, asigna a nuestro país una tasa cruda/100.000 h de 1862,5 y ajustada a población mundial de 1175,4. Como podemos comprobar las cifras son muy próximas a las nuestras (2.034 y $1295,2 / 100.000$ respectivamente). 
La mayor prevalencia de cáncer en el sexo masculino no es hecho habitual en la mayoría de los países ${ }^{1}$, pero es un dato permanente en los estudios referentes a nuestro país. Así, como hemos visto, los datos de la OMS asignan a España unos 30.000 casos de cáncer más en hombres que en mujeres ${ }^{1}$. También hemos comprobado que en nuestra AS se han producido un $25,6 \%$ más casos de tumores de nuevo diagnóstico en varones que en mujeres (datos de admisión del Hospital General). Las diferencias fundamentales, además de aquellas ligadas al sexo, están en relación con los tumores teóricamente relacionados con el consumo de tabaco (de aparato respiratorio y urinario). Dado que su consumo era claramente diferente entre hombres y mujeres en años previos en nuestro país, asumimos este hecho como uno de los factores fundamentales también en nuestra AS, aunque no debemos descartar otros posibles factores etiológicos, como podrían ser los laborales.

Estos mismos factores pueden influir también en las diferencias entre el medio urbano y el rural; como vemos en las tablas 2 y 3 las prevalencias, una vez corregidas por edad, son más altas prácticamente en todas las localizaciones en el medio urbano y comarcas periurbanas. Y precisamente el grupo de tumores ligados al consumo de tabaco y/o factores laborales tienen también una alta representación en las zonas urbanas y en aquellas comarcas con mayor industrialización (Ciudad y Campiña).

Aunque los estudios de prevalencia no suelen presentar datos desglosados en función del medio, en algún caso se publican datos de las grandes ciudades ${ }^{11,12}$ con mayor prevalencia de la mayoría de las localizaciones en el medio urbano, una vez realizado el ajuste por edad. No obstante, el diseño de nuestro estudio no permite inferir explicaciones etiológicas respecto a las diferencias de tasas en función del lugar de residencia. En este sentido, tal y como aducen otros autores, es posible también que el medio urbano produzca una mayor atracción a los pacientes diagnosticados de cáncer debido a las diferentes condiciones de vida, recursos sociales y medios asistenciales, que hacen que estos pacientes se concentren alrededor de los lugares mejor dotados y más accesibles a dichos servicios ${ }^{30,31}$. Otra posibilidad es que la mayor disponibilidad de medios diagnósticos y accesibilidad a las pruebas de cribado en el medio urbano produzcan un adelanto en el diagnóstico o una detección de tumores que podrían haber evolucionado asintomáticamente y que en el medio rural no habrían llegado al diagnóstico o habrían sido diagnosticados más tardíamente ${ }^{32,33}$.

La proximidad a las Centrales Nucleares se ha considerado, tanto a nivel social como sanitario, como posible factor de riesgo para padecer cáncer; sin embargo, al margen de las situaciones excepcionales de fallos de seguridad, no se ha demostrado concluyentemente un incremento de casos o mortalidad atribuibles a este factor ${ }^{34}$, ni siquiera en la situación, potencialmente más relacionada, de leucemias y otros cánceres en niños ${ }^{35}$. Algunos estudios han publicado en nuestro país datos de mayor mortalidad por tumores hematológicos, específicamente leucemia en la proximidad de algunas centrales nucleares y mieloma en la proximidad de la central de Zorita de los Canes en Guadalaja$\mathrm{ra}^{18,19}$. También una reciente investigación en nuestra Area de Salud encuentra una tendencia al incremento de incidencia de tumores a medida que disminuye la distancia a la Central Nuclear de Trillo y de modo específico en tumores más radioinducibles ${ }^{16-17}$. Sin embargo, se reconoce la dificultad de atribuir una relación causal a la proximidad a las centrales nucleares y la necesidad de correlacionar los casos con datos objetibables (dosimetrías, otros factores de riesgo, etc.), por lo que se consideran necesarios, y actualmente se están planteando, nuevos estudios específicos en nuestro país.

Nuestro trabajo tampoco demuestra diferencias significativas referidas a casos pre- 
valentes en relación con la proximidad a centrales nucleares, si bien un estudio de prevalencia de punto como el nuestro no permitiría en ningún caso verificar esta hipótesis etiológica; así, aunque las cifras de cáncer broncopulmonar prevalente apuntan hacia un mayor riesgo, del mismo modo que encuentra López Abente en relación con la mortalidad $^{19}$, no podemos inferir explicaciones de posible relación causal en este sentido y sería necesario realizar un ajuste en función de otros factores y específicamente del consumo de tabaco.

En relación con las diferencias entre comarcas, los resultados parecen ser reflejo de las que se producen en el medio urbano y periurbano. Unicamente hemos encontrado mayores prevalencias de cáncer de tiroides en la mujer en las dos comarcas más deprimidas y montañosas del Area de Salud. Aunque la casuística es escasa, parece un dato científicamente coherente, siendo conocido el hecho de que el defecto de yodo en la dieta, habitual en zonas montañosas, puede ser factor de riesgo para cáncer y que las zonas bociógenas pueden tener mayor incidencia de cáncer de tiroides ${ }^{36,37}$.

Finalmente queremos destacar el hecho de haber obtenido datos de cada una de las ZBS; esto ofrece la posibilidad de disponer de un registro de la patología neoplásica en cada EAP, que debería ser actualizado para no perder esta importante fuente de información. Tanto por las repercusiones permanentes del diagnóstico de cáncer, como por los recursos que consume su adecuada atención, los profesionales de Atención Primaria deben tener claramente identificada su población con enfermedad neoplásica. Es reconocida la importante carga psicológica del diagnóstico ${ }^{38}$ y que el $34 \%$ de los pacientes ancianos con cáncer necesita cuidadores durante más tiempo y a mayor coste que los ancianos sin dicho diagnóstico ${ }^{39}$.

A su vez, la existencia de datos de cada EAP facilitará la implantación definitiva del
Registro de Tumores, tal y como se produce en otras Areas o provincias. Creemos que esta posibilidad es más importante que los datos en sí y que las diferencias entre centros no tienen transcendencia epidemiológica, dada la inestabilidad de los mismos en poblaciones pequeñas; pensamos que siguen una distribución bastante homogénea y que dichas diferencias vienen dadas por la propia estructura poblacional y causas debidas al azar, salvo las ya comentadas en relación con el medio. Cabe señalar que los valores extremos corresponden a las zonas menos pobladas en las que variaciones mínimas en los casos (por incidencia o por grado de declaración) tienen un impacto importante en las tasas.

Como conclusión, creemos que la prevalencia de cáncer es ligeramente superior en varones que en mujeres $y$, una vez, corregida por edad, mayor en el medio urbano que en el rural. De modo general, no podemos atribuir un mayor riesgo de haber padecido cáncer a la residencia en zonas próximas a las centrales nucleares ni en las distintas comarcas de nuestra Area de Salud, salvo en función del carácter urbano o rural de las mismas. Es necesarios otro tipo de estudios para conocer el impacto real de las centrales nucleares y para conocer si la residencia en zona urbana es factor etiológico en relación con el cáncer o es consecuencia del padecimiento de éste.

\section{AGRADECIMIENTOS}

A todos los profesionales de Atención Primaria de Guadalajara que han participado en el estudio y a los servicios de admisión de los centros asistenciales que nos han facilitado los datos. A Mar Pérez y Blanca Huetos por su colaboración en la obtención y procesamiento de los datos.

\section{BIBLIOGRAFIA}

1. Ferlay J, Bray F, Pisani P, Parkin DM. Globocan 2000. Cancer Incidence, mortality and prevalence 
worldwide (2000 estimates). IARC Cancer Base $\mathrm{n}^{\circ}$ 5. Lyon: IARC Press;2001.

2. Instituto Nacional de Estadística. Defunciones según la causa de muerte 1999. Base de datos INE base (consultado 22 de Enero de 2002). Disponible en: www.ine.es.

3. Instituto Nacional de Estadística. Encuesta de morbilidad hospitalaria 1999. Base de datos INEbase (consultado 22 de Enero de 2002). Disponible en.www.ine.es.

4. Berrino F, Cascinelli N. Cancer prevalence. What for? Tumori 1999; 85:414-7.

5. Capocaccia R; De Angelis R. Estimating the completeness of prevalence based on cancer registry data. Stat Med 1997; 28: 425-40.

6. Zanetti R, Micheli A, Rosso S, Sant M. The prevalence of cancer: a review of the available data. Tumori 1999; 85: 408-13.

7. Feldman AR, Kessler R, Myers MH, Naughton D The prevalence of cancer. N Eng J Med 1986; 315: 1394-7.

8. Hewitt M, Breen N, Devesa S. Cancer prevalence and survivorship issues: analyses of the 1992 National Health Survey. J Natl Cancer Inst 1999; 91: 1480-6.

9. Polednak AP. Estimating the prevalence of cancer in the United States. Cancer 1997; 80: 136-41.

10. Colonna M, Hedelin G, Esteve J, Grosclaude P, Launoy G, Buemi A et al. National cancer prevalence estimation in France. Int J Cancer 2000; $87: 301-4$.

11. Teppo L, Hakama M, Hakulinem T, Lehtobeb M. Saxen E. Cancer in Finland 1953-1970: Incidence, Mortality and Prevalence. Acta Pathol Microbiol Scand [A]. 1975; SUPPL 252: 1-79.

12. Tulinius H, Storm H, Pukkala A, Andersen A, Ericson J, Cancer in the nordic countries 1981-86. Acta Path Microbiol Immunol Scand 1992; APMIS Suppl. 1992; 31: 1-194.

13. Morales Suárez-Varela JM, Domínguez Lillo G, Llopis González A, Ferrándiz Ferragut J. Estudio comparativo de la mortalidad por cáncer según la actividad laboral de la población. Rev Clin Esp 1994; 194: 464-71.

14. Pearce N, Teif JS. Epidemiologic studies of cancer in agricultural workers. Am J Ind Med 1990; 18: 133-48

Rev Esp Salud Pública 2004, Vol. 78, N. ${ }^{\circ}$
15. Bayo A. Incidencia de cáncer en la provincia de Guadalajara 1995-1998;Madrid; 2001

16. Viana Gil D. Estudio epidemiológico de la asociación entre patología oncológica y grado de proximidad residencial a las Centrales Nucleares de la provincia de Guadalajara [tesis doctoral]. Madrid: Universidad de Alcalá; 2000.

17. Silva-Mato A, Viana D, Fernández-San Martín MI Cobos J, Viana M. Cancer risk around the nuclear plants of Trillo and Zorita (Spain). Occup Environ Med 2003; 60: 521-7

18. López -Abente G, Aragonés N, Pollán M, Ruiz M, Gandarillas A. Leukemia, Lymphomas and myeloma mortality in the vicinity of nuclear plants and nuclear fuel facilities in Spain. Cancer Epidem Biomar 1999; 8: 925-34.

19. Lopez-Abente G, Aragonés N, Pollán M. Solid tumour mortality in the vicinity of uranium cycle facilities and nuclear power plants in Spain. Environ Health Perspect 2001; 109: 721-9

20. Alonso Gordo JM, Barcena Marugán A, Jiménez del Val D, Palacios Rojo JJ, Royo Sánchez C, Urbina Torija J y Grupo PREVALCAN de Guadalajara. Prevalencia de cáncer en el Area de Salud de Guadalajara. Aten Primaria 2003; 32:150-7

21.- Dirección General de Protección Civil. Plan de Emergencia Nuclear de Guadalajara - PENGUA. Madrid: Protección Civil; 1991.

22. Muir CS. Classification. Cancer Incidence in Five continents, Vol 6, Chapter 3. Lyon: IARC; 1992.

23. Dos Santos Silva I. Epidemiología del cáncer: principios y métodos. Lyon Agencia Internacional de Investigación sobre el cáncer;1999.

24. Avellana E, Mendive JM, Martí J, Cots JM, Ciurana R, Espel C et al. Registro de tumores en un centro de asistencia primaria. Aten Primaria 1992; 9: 137-43.

25. Botella Cubells S. Estudio de la tasa de prevalencia instantánea del cáncer en una población de referencia. Medicina Militar 1993; 49, 2: 159-62.

26. Adami HO, Gunnarsson P, Sparén P, Eklund G. The prevalence of cancer in Sweden, 1984. Acta Oncol 1989; 28 (4): 463-70.

27. Mariotto A, Dally LG, Micheli A, Canario F. Verdecchia A. Cancer prevalence in Italian regions with local cancer registry. Tumori 1999; 85: 400-7. 
28. Micheli A, Francisci S, Krogh V, Rossi AG, Crosignani $\mathrm{P}$ and the ITAPREVAL Working group. Cancer prevalence in italian cancer registry areas: the ITAPREVAL study. Tumori 1999; 85:309-69.

29. Micheli A, Mugno E, Krogh V, Quinn MJ, Coleman M, Hakulinem $\mathrm{T}$ et al. Cancer prevalence in European registry areas. Ann Oncol 2002; 13: 84065.

30. Melton LJ, Brian DO, Williams RL. Urban-rural differential in breast cancer incidence and mortality in Olmsted County, Minnesota, 1935-1974. Int J Epidemiol 1980, 2: 155-8.

31. Sharier M, Ahnen DJ. Colorectal cancer survival in Europe: the Will Rogers phenomenon revisited. GUT 2000; 47: 463-4

32. Schootmann M, Fuortes LJ. Breast and cervical carcinoma: the correlation of activity limitations and rurality with screening, disease incidence and mortality. Cancer 1999; 86: 1087-94.

33. Muldoon JO, Schootman M, Morton RF. Utilisation of cancer early detection services among farm and rural nonfarm. J Rural Health 1996; 12 (4 Suppl): 321-31.

34. Hattchouel JM, Laplanche A, Hill C. Cancer mortality around French nuclear sites. Ann Epidemiol 1996; 6: 126-9.

35. Kaatsch P, Kaletsch U, Meinert R, Michaelis J. An extended study on childhood malignancies in the vicinity of German nuclear power plants. Cancer Causes Control 1998; 9: 529-33

36.- World Cancer Research Fund in association with American Institute for Cancer Research. Food,
Nutrition and the Prevention of Cancer: a Global perspective. Washington: American Institute for Cancer Research;1997. P. 327.

37. Galanti MR, Sparen P, Karlsson A, Grimelius L, Ekbon A. Is residence in areas of endemic goitre a risk factor for thyroid cancer? Int J Cancer 1995; 61: 615-21.

38. Little M, Sayers EJ, Paul K, Jordens CF. On surviving cancer. J R Soc Med 2000; 93:501-3.

39. Hayman JA, Langa KM, Kabeto MU, Katz SJ, DeMonner SM, Chernew ME et al Estimating the cost of informal caregiving for elderly patients. J Clin Oncol 2001; 19: 3219-25.

\section{Anexo 1}

El Grupo PREVALCAN está formado por las siguientes personas: Coordinadores del estudio: Alonso Gordo JM, Barcena Marugán A, Jiménez del Val D, Palacios Rojo JJ, Royo Sánchez C, Urbina Torija J. Participantes y Centro de Salud al que pertenecen: Anuncibay Ibáñez LA (Checa), Chavida García F (Brihuega), Chamorro González AL (Maranchón), Delgado Delgado P (Horche), Díez Andrés ML (Cervantes-Guadalajara), Díez Cantarero A (Balconcillo-Guadalajara), Díez Morán R (Yunquera), Estal Lizondo J (Villanueva), García Lacunza MA (Delegación de Sanidad), Isasia Ballesteros J (El Pobo), López Galindo P (El Casar), Luengo Rojo S (Molina), Martín Millana JC (Cifuentes), Millán Catalán M (Mondéjar), Parrilla Rubio M (Hiendelaencina), Prats Pérez C (La Campiña-Marchamalo), Santos Altozano C (Alamín-Guadalajara), Santiago González C y Tejedor Montero F (Azuqueca) y Torres Buisán L (Atienza). 